Voix et Images

voixetimages

\title{
Anne Hébert ou le désert du monde
}

\section{Lucille Roy}

Volume 7, numéro 3, printemps 1982

Anne Hébert

URI : https://id.erudit.org/iderudit/200343ar

DOI : https://doi.org/10.7202/200343ar

Aller au sommaire du numéro

Éditeur(s)

Les Presses de l'Université du Québec

ISSN

0318-9201 (imprimé)

1705-933X (numérique)

Découvrir la revue

Citer cet article

Roy, L. (1982). Anne Hébert ou le désert du monde. Voix et Images, 7(3), 483-503. https://doi.org/10.7202/200343ar

Ce document est protégé par la loi sur le droit d'auteur. L'utilisation des services d'Érudit (y compris la reproduction) est assujettie à sa politique d'utilisation que vous pouvez consulter en ligne.

https://apropos.erudit.org/fr/usagers/politique-dutilisation/ 


\title{
Anne Hébert ou le désert du monde
}

\author{
par Lucille Roy, Dawson College; Montréal
}

L'artiste n'est pas le rival de Dieu. II ne tente pas de refaire la création. (...) Il sait le prix du jour et de la lumière.'

De toutes les puissances élémentaires qui dominent l'univers poétıque d'Anne Hébert, de ces forces qui, s'intégrant à la vie humaine, finissent par l'orienter dans un sens précis, il n'en est pas de plus captivante que la lumière. Du début à la fin de l'œuvre, celle-ci s'impose par sa présence ou son absence: car tout ici est appel de clarté, recherche d'un jour qui, à des degrés différents d'intensité, façonne la vie de l'homme. La nuit elle-mème n'est que le vide que creuse l'absence du soleil : elle participe à la quête d'une lumière essentielle dont l'importance vitale se manifeste déjà dans les Songes en équilibre:

De la terre

Où je vois la lune

Aux bleus cratères

Je crie:

Délivrez mon âme

Des paysages lunaires

Que le soleil n'atteint plus ! ${ }^{2}$

\section{A. L'envoûtement et l'oppression}

Mais il faudra attendre Kamouraska pour pouvoir mesurer toute la puissance envoūtante de cette lumière extraordinaire qui ne semble pas moins cruelle pour le corps que pour l'esprit. Antoine Tassy, dans son long cheminement vers la mort, est victime de rayons trop intenses qui l'attirent et le retiennent auprès d'une fenètre. Élisabeth elle-mème, ne risque-t-elle pas de devenir prisonnière de cette même clarté, "prise au piège" d'une force violente et captivante?:

II y a trop de soleil dans cette maison aussi. Antoine ne tente aucun geste pour se retirer de la fenêtre où il se tıent debout, exposé en pleine lumière. Ses yeux rougis ne clignent pas. On dirait qu'il se soumet lui-même au supplice de la lumière. 
Un long rayon traverse la pièce, m'atteint de plein fouet. Je suis prise au piège de la lumière à mon tour. Je détourne la tête. ${ }^{3}$

En effet, si Antoine est asupplicié " par cette lumière inhumaine, au point de ne plus pouvoir se protéger, en fermant les yeux. Élisabeth tente en vain de se libérer. Les images du fouet et du piège, en soulignant la violence captivante de la puissance cosmique, rendent du même coup insignifiant tout geste de révolte.

La lumière paraît s'imposer d'abord par sa densité ou son poids. Elle opprime et bouleverse parce qu'elle écrase, causant un état de faiblesse [ou] de fatigue. Toujours située au-dessus de l'être, elle semble tomber de très haut, telle une matière lourde qui s'abat sur la vie pour entraîner la mort: "La lumière», dit Élisabeth, "de plus en plus violente, tombe maintenant en faisceaux du plafond, au-dessus de mon lit. Je pourrais me croire sur une table d'opération. ${ }^{4}$ Liés ensemble comme des gerbes de blés, ces rayons étrangement denses pèsent de tout leur poids sur un corps affaibli, passif et angoissé.

Dans « La ville tuée», la lumière est rapprochée d'un vent destructeur, dont le poids étouffe et dessèche le pays. La lumière, en effet, est la seule puissance cosmique présente au début du poème: c'est en pleine lumière que s'étanche le marais, qu'on bannit tous sentiments et que la vie se réduit à l'os. Le cœur lui-même, cet "oiseau de proie" dont le sens nous sera dévoilé dans "Le Tombeau des rois* 5 , est capturé et crucifié par cette même clarté néfaste. Et c'est alors que descend le vent, avec son poids accablant, croissant sur l'âme comme un jugement dernier:

On étancha le marais, l'oiseau de proie fut capturé, toutes ailes déployées, le plus doux d'entre nous nous assura qu'il le ferait dormir en croix sur la porte

$(\ldots)$

La main droite de chacun fut posée à plat sur le ciel afin de vérifier chaque jointure et la précision des os dans la lumière

(...)

Un vent lourd s'abattit sur toutes choses. C'était le jugement au comble de lui-mème croissant sur nous, régnant à perte de vue ${ }^{6}$.

La main "posée à plat", le corps "étendu", les diverses allusions au sommeil, tout contribue dans ce poème à créer l'image d'une vie complètement écrasée et étouffée par une force supérieure - que la poète identifie à une lumière ayant toute la puissance et la densité du vent.

Un autre poème d'Anne Hébert nous révèle la force opprimante de la lumière. Le "ravin de fatigue" oú le sujet du poème se sent rouler jusqu'au fond n'est pas creusé par l'eau, mais par une lumière intense, bleue ou blanche qui émet un “ fracas d'ivoire ”. Dure et écrasante, cette clarté insolite, 
toujours située au-dessus de l'ètre, est dotée d'une voix qui témoigne de sa puissance destructive. Son grand cri desséchant pénètre l'oreille du poète comme des grains de sable, tandis que son poids abîme de plus en plus le corps:

Rouler dans des ravins de fatigue

Sans fin

Sans reprendre haleine

(...)

Fracas d'ivoire à mi-voix

Contre notre oreille pleine de sable

Bleu du ciel

Grand cri de la lumière au-dessus de nous. ?

Car nous voyons dans cet enchaînement de consonnes liquides et sifflantes, moins l'essoufflement d'une passion quelconque (comme l'affirme PierreHervé Lemieux $)^{8}$, qu'une espèce de glissement progressif vers le bas, la continuité de l'action oppressive de la lumière et de la chute. Rien, en effet, ne nous autorise à supposer ici la présence d'un partenaire, le "nous" étant employé, au dire du poète elle-même, pour objectiver son mal, opérant une distanciation salutaire par rapport à sa propre personne. ${ }^{9} D^{\prime}$ ailleurs, la jeune fille du poème est prise dans ses propres cheveux, la fière qu'elle éprouve étant presque toujours, chez Anne Hébert, symbole de solitude et d'emprisonnement. 10 Ainsi, ce qui produit dans ce texte la chute du sujet ne nous semble nullement un abandon total à l'amour, mais bien au contraire, l'effet d'une action externe - de cette lumière accablante dont la densité et le poids écrase et épuise le cceur. Comme l'aivaire» et le "sable», la clarté de ce jour est dure et desséchante. Singulièrement lourde, elle ne peut que précipiter. l'être dans l'abìme, le rattachant au sol.

On pourra donc conclure que la lumière qui se manifeste dans Le Tombeau des rois a une puissance captivante, écrasante et néfaste. Son intensité dessèche; et son poids, effet apparent de sa densité, pousse graduellement l'être dans l'abîme. Force accablante, capable de ronger jusqu'au cceur de la vie, étouffant toute émotion, cette lumière opaque et dense, dénuée de toute transparence. est essentiellement "pourrie»: "La touche limpide de la lumière est gâtée à jamais pour moi ", s'écrie déjà François dans Le Torrent "

Dans l'œuvre d'Anne Hébert, un lien étroit s'établit entre la puissance envoùtante de la lumière et l'intensité du regard, comme si celui-ci participait directement de la force cosmique. Ce n'est guère par hasard que les yeux de tant de personnages hébertiens sont jaunes, ou que le regard de Claudine, d'Amica, d'Aurélie et de sœur Julie ressemble étrangement à celui d'un chat. "Le chat établit une continuité entre tous les regards", écrit Albert Legrand au sujet du Torrent '2. Le chat, comme la femme chez Anne Hébert, semble recevoir de la lumière du jour la clarté de sa vision - une force captivante et opprimante. Ainsi que les rayons du soleil, le regard enflammé du chat et 
d'Amica vise. le monde avec intensité, le feu n'étant apparemment ic̉i qu'une forme plus concentrée de la lumière:

Un chat rôda alors aux environs, dit François $(\ldots)$

La bête consciente et hors d'atteinte continuait sur moi son fixe regard d'éternité (...)

Amica a les mêmes yeux que ce chat. Deux grands disques en apparence immobiles, mais qui palpitent comme la flamme. ${ }^{13}$

L'éclat jaune de ce regard, qui évoque toute l'ardeur et la violence du soleil hébertien, attire et retient le regard d'autrui, imposant à celui-ci une vision humiliante de son être. Autant, dans Le Tombeau des rois, l'âme du poète se sentait prisonnière de la lumière jusqu'à l'épuisement et la chute, autant l'œil de sceur Julie des Enfants du sabbat fixe, restreint et condamne l'aumônier à sa pitoyable nullité. Si dans le poème précité. l'intensité du jour conduisait le sujet a l'abîme, le regard de. Julie, "couleur de safran», opprime la vie de l'aumônier en la "crucifiant m:

Trop tard, l'œil de sœur Julie se pose un instant sur l'aumônier, parfaitement étale, vide, couleur de safran.

Incapable de bouger ni de baisser les yeux, l'aumônier supplie Dieu, tout bas, de briser l'air entre lui et sœur Julie, afin que se détache de lui ce regard qui l'envoûte et le cloue à sa chaise pour le crucifier. ${ }^{14}$

Nous retrouvons chez Claudine, dans Le Torrent, cette même puissance hypnotique du regard, si semblable dans l'ceuvre d'Anne Hébert à l'envoûtement de la lumière. L'emprise de Claudine sur Francois s'exerce surtout par la communication silencieuse qui s'établit entre le regard de la mère et celui du fils, l'un lançant des aflammes", l'autre comme paralysée de stupeur et d'admiration. C'est ainsi sous sa forme la plus ardente que se révèle ici la présence de la lumière, celle-ci se manifestant continûment dans le regard intense de Claudine qui s'impose à François et l'humilie:

Et. c'est mes yeux égarés retenus dans les siens, dit-il, que se déroula tout l'entreprise. J'étais paralysé, magnétisé par la grande Claudine (...)

Ses yeux lançaient des flammes. Tout son être droit, dressé au milieu de la pièce, exprimait une violence qui ne se contenait plus, et qui me figeait a la fois de peur et d'admiration. ${ }^{15}$

De même, le regard fixe d'Amica accable et blesse François simultanément, "pesant" sur son sommeil d'un apoids étrange" :

(Amica) m'observe, tendue, prête à fuir à la moindre alerte. A la vérité, je sens son regard, même sous ma paupière fermée. Il pèse sur mon sommeil, de tout son poids étrange. C'est lui qui me réveille, à force de concentration. Cela ressemble à de l'hypnotisme. ${ }^{16}$

Enfin, dans Kamouraska, l'auteur associe directement la puissance de la lumière à la fixité du regard. Captivés par le soleil qui les retient à la fenêtre, 
se soumettant " au supplice de la lumière», les yeux d'Antoine n'en demeurent pas moins grands ouverts et lucides. Ces yeux « rougis $*$ de lumière, qui pourtant ne clignent pas 17, fixent ensuite Elisabeth avec violence et stupeur: “Antoine soudain est debout en face de moi qui me regarde. Cette stupeur sans borne. Son dernier regard. Trop de lumière * 18 . Chez Elisabeth aussi, la lumière détient ce pouvoir extraordinaire sur le regard. L'astreignant à l'«attention la plus stricten, à une clairvoyance absolue, le soleil qui envahit la chambre de Léontine constitue un supplice qui condamne l'héroïne à la vérité. Associée au regard jaune d'Aurélie, cette clarté lourde et intense viole les secrets du drame de Kamouraska:

Une lumière extraordinaire entre à flots, déferle jusque sur le lit (...) Aurélie marmonne, tout en préparant mes vêtements pour la journée:

- On ne peut pas toujours vivre dans la noirceur. II faut ce qu'il faut. Les grandes scènes de votre vie s'en viennent, Madame. C'est en pleine clarté qu'il faut les revivre (...)

L'éclair jaune de son $\boldsymbol{\infty}$ il, entre les cils (...)

Ce n'est pas tant la netteté des choses en soi qui me bouleverse. Mais je suis forcée (dans tout mon ètre) à l'attention la plus stricte. Rien ne doit plus m'échapper (...) Tant de précision. ${ }^{19}$

Au début de cette cuvre, ce regard fixe et oppressif demeure pourtant la seule puissance qui rattache l'homme au monde, maintenant l'individu en communication constante avec sa propre vie et celle des autres. L'homme ne peut exister ici que dans la mesure où ses yeux sont ouverts et vifs: de là viennent toute sa vitalité et son dynamisme. Dans la maison à moitié inhabitée qu'est la personnalité de Claudine Perreault, la vie ne demeure que dans la clarté du regard: "Seul l'éclat des yeux ne se retirait pas tout à fait, ainsi que les restes d'une fête dans une maison déserte" 20 . Et lorsque la jeune fille des Songes en équilibre se voit aveuglée par la pluie et le brouillard. c'est sa vie entière qu'elle remet en doute.

Pas bien sûre de m'être jamais connue,

Aveugle, errante.

Perdue sous la pluie

Perdue sous la brume ${ }^{21}$.

Ainsi, dans «La robe corail», les yeux d'Émilie, grands ouverts mass vides, témoignent d'un être qui n'existe qu'à l'état virtuel. Ils sont démesurément "grands", parce que, "sans présence ni rêve», ils n'expriment que l'absence de tout contact avec la vie ${ }^{22}$. Comme l'écrit René Juéry, les yeux d'Émilie. "métonymiques de son corps, sont des sphères difficilement perçues parce que vide de matières ${ }^{23}$.

\section{B. Le rayonnement et le viol}

La présence du regard - clairvoyance qui naît d'une interaction dynamique entre les yeux et la lumière externe, assure. chez Anne Hébert, un lien 
concret avec le monde. Cette lumière intense et captivante, en s'imposant à l'être, fait naître aussitôt le désir. «Ce désir que j'avais augmentait de jour en jour $x$; dit François en partant à la recherche d'autrui sur une route ensoleillée 24 . Et plus' tard, il ajoutera: "Le désir de la femme m'a rejoint dans le désert ${ }^{25}$. L'image du désert. évoquant tout l'aspect desséchant de la lumière hébertienne, rappelle trop vivement cette route «unie * et «ans âme», étendue «au soleil» où autrefois François s'est aventuré en quête d'un visage humain ${ }^{26}$. A ce sujet. Albert Legrand pourra écrire: $\propto$ Toute cette prestigieuse et terrifiante aventure n'a-t-elle pas commencé sous le signe du désir ? ${ }^{27}$

La naissance soudaine du désir sous l'emprise de la lumière marquera la fin des songes:

Ce désir qu'on croyait mort, renaît;

Et le songe en équilibre

Est tout désarticulé ${ }^{2 B}$.

A partir du Torrent, l'intensité de la lumière conjuguée au regard lie le moi au monde de façon brutale, presque inhumaine. Ce désir qui soutient la quête ardente de François n'est pas une douceur; il demeure aimpitovable s jusqu'a la fin de l'ceuvre ${ }^{29}$. Tout se passe comme si l'homme était agi par une force cosmique qui le dépassait et l'opprimait. Une telle exigence face au réel aura pour corollaire le rejet de tout ce qui, dans les poèmes antérieurs, planait au-dessus de la vie, demeurant léger et fuyant, tels la danse et le songe. "Le mouvement (des Songes en équilibre ), écrit Pierre Pagé, «est danse, arabesque, fusion avec le monde aérien ${ }^{30}$.

Vers 1944, date de la parution d'un poème intitulé a L'infante ne danse plus». la danse est perçue par le poète comme la suprême absence. L'être qui danse ne peut plus exister chez Anne Hébert, parce qu'il avait peu de contact avec le réel, parce qu'il ne voyait rien. Échappant totalement à l'envoûtement de la lumière, sans présence ni poids, faisant de la terre un simple tremplin pour bondir dans l'au-dela, il n'était que mouvement: «Rien que le geste pur», écrit l'auteur de «L'ange de Dominiquen, «rien que toute sa valeur rendue au geste ${ }^{31}$. La danse, qui se situe ainsi aux antipodes du regard aigu. opprimant et liant, était désirée par le poète des Songes... comme une fuite de la réalité, un pur artifice:

O être délivré

par un pas libre et parfait

un pas de danse! 32

Anne Hébert, d'ailleurs, ne tardera pas à s'en rendre compte. La danse extraordinaire qu'Ysa apprend à Dominique entraîne aussitôt la mort de celle-ci ${ }^{33}$. Et la liberté illusoire du bond disparait complètement de cette ceuvre. lorsque «l'infante ne danse plus "34. C'est alors que l'auteur, se vouant dorénavant à la clairvoyance la plus astreignante, fait dire a Pierre de $\alpha$ L'arche de midi»:

Plus d'abri ni de paix possible une fois l'aventure de la terre apprise. Je ne suis plus libre. Je ne serai plus jamais libre ${ }^{35}$. 
C'est par les mains que la lumière hébertienne noue un lien concret entre le sujet et le monde. Dans sa thèse sur l'aliénation dans l'couvre d'Anne Hébert, Francis Macri affirme: “Les mains constituent (...) le premier geste vers l'extérieur, vers l'espace illimité du monde» ${ }^{36}$. Mais ce que ce critique néglige de mentionner, c'est l'effet extraordinaire que la lumière hébertienne produit sur ces mains. Dans un poème du Tombeau des rois, les mains du sujet sont rattachées directement à la lumière, au point où le soleil « grave» sur ces mains les "signes du monde» lui-même. En effet, les mains servent ici de « miroir * à la lumière du jour qui, en s'y reflétant, les colore, les marque et les oriente vers autrui. Ainsi le sujet du poème, subissant sur son corps la force rayonnante du soleil, « ouvre» largement ses mains à la vie, malgré la douleur qui parait résulter de leur confrontation avec le réel. Ainsi offertes à la lumière. ces mains finissent par rayonner elles-mêmes comme un soleil, éclairant le monde extérieur:

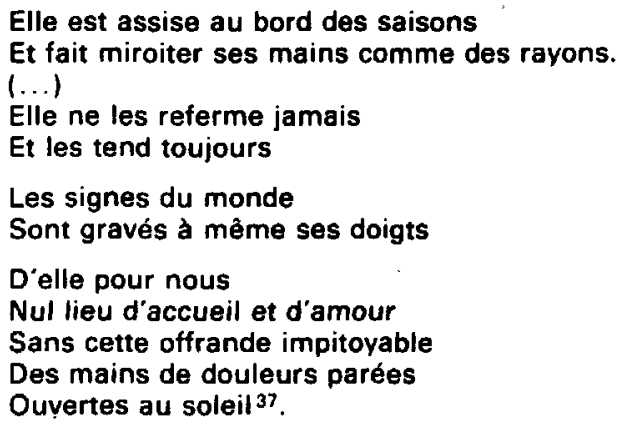

Il est intéressant de noter à quel point la lumière qui se reflète sur ces mains demeure captivante et lourde. En rattachant celles-ci au monde, la clarté du jour les orne de abagues" «accablantes" et "massives», capables de "graver" jusque dans la chair les signes du monde. Dans son étude sur Le Tombeau des rois, Pierre-Hervé Lemieux souligne le comportement étrange de cette femme qui demeure fascinée et comme obsédée par l'éclat du soleil sur ses mains. indiquant en même temps la place privilégiée que le poète accorde à ce thème dans l'édition originale du recueil ${ }^{38}$. Ce qui est sûr, c'est que cette offrande des mains au monde, - don qui ressemble plutōt à un sacrifice, tellement la jeune fille du poème paraît prisonnière de l'effet rayonnant d'une lumière aussi douloureuse, - constitue un tournant principal dans l'œuvre d'Anne Hébert. Car tout jusqu'ici, dans la poésie des Songes... était évasion du monde par le rêve et repliement sur soi. Les mains du poète, "incolores" parce que non encore soumises à la lumière, ne se tendaient vers personne:

Ces mains jamais déliées,

Ces gestes à jamais repliés.

Ces gestes que la terre

Pas plus que l'éther 


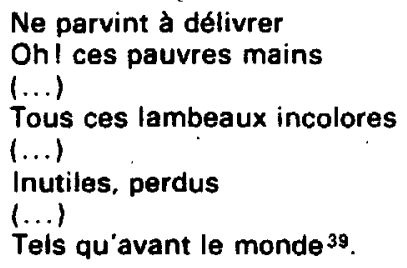

A partir du Torrent et du Tombeau des rois, l'être hébertien ne sera donc vivant que dans la mesure où, sous l'effet d'une lumière et d'un désir ardents. ses yeux demeureront ouverts et ses mains se tendront vers la vie. Chez Catherine des Chambres de bois, la menace de la mort se manifestera d'abord par le retrait des mains et le refus d'entrer en contact avec les objets et les êtres: «Les premières, ses mains vinrent à manquer, refusant tout contact avec les choses et les gens de cette maison ${ }^{40}$. Et nous pourrons suivre l'évolution de ces mains tout le long de l'ceuvre d'Anne Hébert, depuis ce premier contact quasi involontaire avec la lumière du jour jusqu'à leur intégration complète au monde.

Un autre poème du Tombeau des rois, intitulé aUn bruit de soie", nous fait voir plus directement encore le rapport quasi organique qui s'établit dans son ceuvre entre la lumière, le regard, les mains et le désir. Sous le soleil de midi, deux amants se cherchent par la seule force de leurs "bras étendus". L'ardeur de leur désir, qui semble participer d'une lumière intense, fait avancer ces «mains avides», comme des «serviteurs" fidèles au cosmos et à la passion du cceur. Les bras ou les mains qui se tendent ainsi vers la vie sont essentiellement agis; ils sont pour ainsi dire propulsés par le regard, la lumière et le désir:

Trop de lumière empèche de voir

$(\ldots)$

Se chercher à travers le feu et l'eau fumée

Nos bras étendus nous précèdent de deux pas.

Serviteurs avides et étonnés

En cette dense forêt de la chaleur déployée Lente traversée ${ }^{41}$.

Commentant ce texte. Pierre-Hervé Lemieux indique l'aspect féminin et doux de la lumière présentée dans la première strophe, malgré "l opacité craveuse" qu'il lui reconnaît dans les strophes subséquentes. Aussi peut-il affirmer: "Le vent est amadoué, il est féminisé par la comparaison avec un «bruissement" de soie. De même pour la lumière, qui ne dévore plus, mais se trouve adoucie et assimilée à cette "soie ${ }^{42}$. Nous croyons au contraire que l'image d'un abruit de soie «, conjuguée au mouvement rapide du vent et rapprochée de l'“éclat dè midi * qui ouvre la strophe suivante, souligne plutôt la célérité et la violence de la lumière, sa chute trop brusque et opprimante sur ce "paysage d'eau" qu'elle fera aussitôt afumer». On ne peut, en effet, se défaire, en lisant ces trois premiers vers, d'une impression de trahison profonde ou de fatalité. La 
lumière étouffante que semble faire avancer l'un vers l'autre les deux amants efface du même coup toute douceur et humanité. Ce lien est brutal, presque cruel.

Mais guère plus cruel que la lumière elle-même. Dans l'univers poétique d'Anne Hébert, les rayons du soleil ont souvent un caractère étrangement aigu. lis ne peuvent rattacher l'homme au monde, semble-t-il, orienter son regard et ses mains, sans percer aussi sa chair. Parfois associée à des griffes ${ }^{43}$ et même à un couteau ${ }^{44}$, cette lumière s'avère étonnement blessante et douloureuse. Lorsque le sujet du poème "Les Mains" expose celles-ci au soleil, la lumière captivante qui les éclaire semble pénétrer jusque dans leur chair. Nous avons vu à quel point les "signes du monde" associés à cette lumière étaient "gravés à même les doigts du sujet comme des "chiffres profonds". Ses mains étaient, pour ainsi dire, sacrifiées à la lumière, dont les rayons blessaient cruellement le corps. Mais c'est surtout dans le poème «Roulër dans des ravins de fatigue " que l'auteur nous fait voir combien la lumière intense qui l'accable est douloureuse et pénétrante. Ici l'image de l'oiseau représente, comme l'écrit Jean-Louis Major ${ }^{45}$, tout ce qui est le plus secret chez le poète. les sensations profondes dévoilées par la lumière. Et lorsque cette vie intime est percée par les rayons du soleil, le cœur se détache du corps, s' “agrafant» au cou du poète. Dans la deuxième strophe, la pénétratıon de la vie intérieure par la lumière prend la forme d'un viol où la poitrine du sujet est "crevée», son corps (cette "cage de bouleau blanc»,) rompu et les secrets de son passé «éventrés". Ainsi l'intensité d'un soleil opprimant finit-elle par ouvrir le corps et le ccur, les exposant impitoyablement au monde:

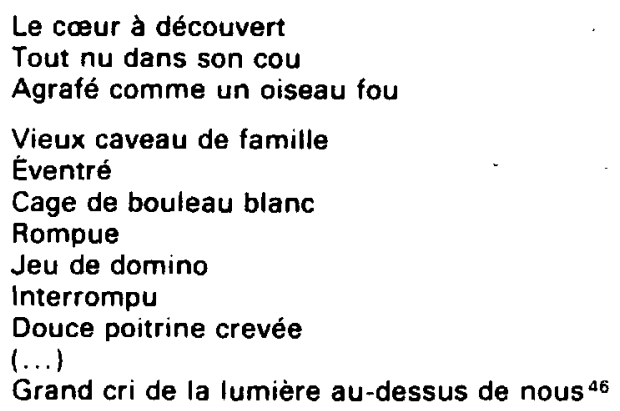

Bien avant que le poète n'ait exprimé toute la cruauté d'une telle lumière. elle semble l'avoir pressentie. Vers 1939, à l'époque où elle écrivait "La robe corail», Anne Hébert avait déjà imaginé des rayons aussi "perçants» qui dissiperaient brutalement un jour le bonheur et les songes d'Emilie:

Mais Émilie a la vague impression que son bonheur ne résistera pas au jour, comme ces vapeurs blanches que perce le soleil et qui flottent. toutes déchirées avant de disparaître ${ }^{47}$. 
Trente ans plus tard, l'héroïne de Kamouraska sera soumise à son tour au supplice de la lumière. Des rayons intenses et aigus, assimilés à des «aiguilies\%, pénètrent l'esprit et le corps d'Élisabeth dans la chambre de Léontine: a La lumière me blesse toujours. Je la sens en aiguilles rouges, brûlantes, sous mes paupières fermées ${ }^{48}$. L'éclair «métallique du regard des témoins qui l'accusent, véritables poignards lui crèvent le cceur ${ }^{49}$, ces "rayons pointus " se durcissent et s'intensifient davantage, se * bardant de feu ». lorsqu'ils pénètrent plus avant dans la chair d'Elisabeth. Et plus cette lumière est intense et opprimante, plus aussi elle paraît dure, blessante et irritante:

Des femmes minuscules en tabliers et bonnets blancs passent à travers mes paupières fermées, s'écrie Élisabeth. Comme des rayons bardés de feu. (...) C'est un phénomène de la lumière. Cela est entré dans la piece avec ces rayons pointus qui déchirent mes yeux, ainsi qu'on extirpe une poussière ${ }^{50}$.

C'est ainsi que l'aube, pour l'héroine de Kamouraska, devient l'image même de l'angoisse. Dans des pages assez pénétrantes, Louisette Lefebvre écrit: "Le présent se décompose sous l'action de la lumière qui devient de plus en plus éclatante 51 .

La dureté et l'acuité de ces rayons néfastes se transmettent aussitôt aux mains qui les reflètent. On se rappellera combien, dans le poème * Les mains", celles-ci étaient prisonnières d'une lumière ardente qui exercait sur elles une action d'ouverture. Or dans alnventaire», qui suit de près le premier poème. les mains du sujet s'avèrent tout aussi tranchantes et brutales que la lumière qui leur sert de force motrice. Dans "un réduit très clair et nu" où se manifestent l'omniprésence et toute la puissance de dévoilement de la lumière, les mains, que le poète associe à une clame vive" et à un "fin couteau", s'insèrent dans la chair nue, pillant les secrets du cour. Plongées à l'intérieur du corps, le a crevant " comme un fruit, la lumière et les mains du sujet violent conjointement le mystère de la vie. l'exposant impitoyablement au monde:

Dans un réduit
Très clair et nu
On a ouvert son coeur
En toute pitié:
Fruit crevé
Fraîche entaille
Lame vie et ciselée
Fin couteau pour suicidés.
Des deux mains plongées
Nous avons tout saisi
Tout sorti ${ }^{52}$.

Alors le sujet se dissocie de ce sang qui lui échappe et abandonne sa chevelure. II rejette son visage nu, son identité propre que, sous l'action de la lumière, ses mains ont en quelque sorte démystifié, en l'exposant à autrui : 
Le sang (qui n'étonne personne)

Rutile

Goutte à goutte

(Quand il brunira

Nous serons loin

Et bien à couvert.)

(...)

La châsse d'or

Que nous avions faite au mystère

Se dresse pillée

Spacieux désert

Sur une table sans pieds,

Son propre visage rongé

Qu'on a aussitôt jeté ${ }^{53}$.

Le poème se développe selon un mouvement de pénétration et d'ouverture progressives. L'espace " réduit » et personnel du début devient à la fin du poème "spacieux désert". Si d'abord la lumière et les mains ne se fixent que sur des objets usuels, tels que des livres, des chiffons et des cigarettes, le viol se poursuit ensuite dans la vie sensible et inconsciente, du "lit défait", à travers "douleurs" et "joies", jusqu'au amystère" de la vie elle-mème ${ }^{54}$. Et le sentiment d'aliénation que le sujet éprouve face à sa vie intime ainsi dévoilée se fait de plus en plus poignant. D'une chevelure qui n'est d'abord que provisoirement "abandonnéen, nous passons ensuite à des joies "bannies", c'est-à-dire inaccessibles, pour aboutir enfin à un visage en voie de destruction, qui est aussitôt « jetém, rendu irrécupérable ${ }^{55}$.

Ce qui amène la troisième image de la constellation: le regard dur et perçant. Aux arayons pointus» d'une lumière qui viole, à ces mains "tranchantes $\nsim$ qui, sous l'emprise du soleil, font froidement l'inventaire de la vie, se conjugue un regard aigu et pénétrant; tel ce «fixe et dur» regard d'Adélaïde Menthe "au visage de pierre ${ }^{56}$. la vision perçante de Lia ${ }^{57}$, mais avant tout le regard captivant d'Amica. Celui-ci, continuant celui de Claudine, ces yeux "enflammés* où paraît se concentrer toute la lumière du jour, dévoile la vie intime de François jusqu'aux profondeurs de son inconscient. C'est le "poids étrange * de ce regard (dont nous parlions plus haut) qui assure ici sa puissance de pénétration:

(Son regard) pèse sur mon sommeil, observe François, de tout son poids étrange (...) A quoi veut-elle en venir? (...)

Elle m'examine quand je dors. Elle me regarde quand je ne me vois plus. Elle peut découvrir dans mes songes les gestes de mon absence, ces gestes enfouis dans les régions les plus obscures de mon être et que le sommeil ressasse à loisir, afin de laisser au matin ce relent amer, juste de quoi nourrir le tourment du jour ${ }^{58}$.

Tout comme le soleil hébertien rattachait au monde les mains de la jeune fille du Tombeau des rois, ainsi la clarté pénétrante du regard livre à 
la société les secrets du moi. La puissance rayonnante de cette lumière se continue dans un regard dont la dureté a essentiellement pour fonction de violer la vie la plus intime pour mieux ensuite l'exposer à autrui. Tous ces regards inquisiteurs sont des regards de témoins, d'observateurs sociaux. Sans sympathie aucune pour la personne épiée. le témoin chez Anne Hébert demeure lucide et cruel, toujours externe, lié au monde: "Je suis nu, dehors. devant cette fille en pillage pour le compte de la policen, s'écrie François 59 :

Témoin, quel mot qui me blesse, m'obsède! Amica est un témoin. Témoin de quoi? Témoin de moi, de ma présence, de ma maison. Cela suffit pour me donner la frousse, comme si je voyais un grand miroir aux images ineffacables retenir mes gestes et mes regards. A aucun prix, il ne faut relâcher mon témoin dans le monde 60 .

Ce même regard dur et inhumain, intégré à la force pénétrante de la lumière, se retrouve plus tard dans Kamouraska et Les Enfants de sabbat. L'“éclat jaune» des yeux d'Aurélie (dont nous avons déjà parlé) se prolonge en quelque sorte dans le regard des trois témoins de Kamouraska, que la clarté intense du jour paraît introduire brutalement dans la vie secrète d'Élisabeth. Ces rayons aigus qui atteignent le cœur de l'héroïne comme des poignards sont en réalité des regards accusateurs visant le centre même de sa vie:

Des femmes minuscules en tabliers et bonnets blancs passent à travers mes paupières fermées. Comme des rayons bardés de feu. (...) Je crois qu'elles font le ménage et disposent des objets sur la commode, comme des pièces à conviction. (...)

Quelquiun d'invisible lance des poignards à la femme, clouée à la planche. Vise son cour ${ }^{61}$.

"Le centre de ma vie, ce désir...", explique plus tard Elisabeth ${ }^{62}$. Le cœur, c'est donc le désir, qui devient ainsi le point de mire de la lumière et du regard hébertiens. Autant la puissance rayonnante du soleil éveille dans cette ceuvre le désir du monde, autant la force de pénétration de cette même clarté, (l'acuité de ces rayons) dévoile ensuite la passion qu'elle a elle-même suscitée. Si le regard d'Amica, participant d'une lumière ardente. pénètre jusque dans la vie inconsciente de François, c'est qu'il révèle chez celui-ci un mal héréditaire (du moins ce que l'auteur appelle un «mal»): l'intensité du désir. François craint surtout dans le fuite d'Amica qu'elle fasse connaître au village que le "mal" l'ait choisi dès le premier souffle de son existence:

Amica m'a vendu (...) Quel surcroît de douleur? (...)

Elle ira dans le monde, répétant qu'elle l'a trouvé ici, que je suis le fils du mal, le fils de la grande Claudine ${ }^{63}$.

Tandis que dans l'esprit d'Élisabeth Rolland, la lueur rouge du soleil, en violant l'intimité de son être sous le regard des témoins, éclaire surtout un "désir. absolu $\approx$, fondamental et essentiel:

Quand un homme et une femme ont rencontré cela, une seule fois, dans leur vie. Ce désir absolu (...). 
II y a un soleil qui bouge au ciel. Une lueur rouge plutôt qui fait semblant d'être le soleil ${ }^{64}$.

Et si le regard d'Aurélie a une telle puissance de fascination sur Élisabeth, c'est que l'éclat vif de ces yeux, évoquant la lumière la plus intense, est le miroir trop fidèle d'une seule et même passion qui les perdra toutes deux:

L'éclair jaune de son œil, entre les cils, dit Élisabeth. Suivent des chuchotements, des clignements d'yeux. Tout un langage incohérent, haletant, impudent et cru. Voix de ma propre solitude ${ }^{65}$.

A ce sujet, Mercedes Telles écrit : «Lumière blessante (...) pour Élisabeth, (Aurélie) éclaire ce qu'elle s'efforce de tenir dans l'obscurité : sa vérité intérieure (...) En rappelant ainsi Élisabeth à sa véritable nature, elle incarne le subconscient de sa maitresse ${ }^{66}$.

Dans Les Enfants du sabbat, søur Julie, dont le regard sait, lui aussi, révéler chez les autres le désir le plus secret, est associée à une lumière aussi brutale. Assise, avec une "brûlure étincelante" aux reins, sur la poitrine du docteur Painchaud, son "œil de chat", qui rappelle les «prunelles phosphorescentes" d'Amica ${ }^{67}$, opprime la conscience du médecin de tout le poids de la vérité la plus cachée de celui-ci. Ce regard a toute la dureté d'un miroir qui reflète fidèlement la passion d'autrui :

L'œil de chat de scur Julie (...) Une pierre d'apparence anodine, en réalité faite pour mirer le cceur le plus secret $(. .$.

Sœur Julie est assise de tout son poids sur sa poitrine, le chevauchant et lui tournant le dos. La brûlure étincelante, au creux des reins, scur Julie se fait de plus en plus lourde (...)

Le poids de sœur Julie se fait oppressant. Tandis que la volupté monte en vagues pour emporter le docteur au-delà de la mort, à la fois redoutée et désirée ${ }^{68}$

Dans ce texte, l'auteur semble avoir donné une forme personnelle à la lumière elle-même. En quelque sorte, le corps de sceur Julie n'est ici quẹ l'intensité et l'acuité de son regard - un regard qui détient à la fois toute l'ardeur de la flamme et une densité minérale. Car cette abrûlure" que l'héroine porte au creux des reins est surtout “étincelante": elle éclaire et dévoile plus qu'elle ne consume, et consume surtout pour mieux dévoiler et opprimer. Comme le soleil chez Anne Hébert, le regard de sceur Julie viole l'inconscient, atteignant. par son intensité et son poids, les désirs les plus secrets.

Mais comment expliquer l'association fréquente que l'auteur fait dans ses cuvres entre le désir et le mal? D'où vient que le "centre" de la vie elle-même prenne souvent un aspect aussi néfaste? II faut se rappeler dans quel contexte social l'homme fait ici l'apprentissage du désir et de la passion. Au moment où l'ceuvre d'Anne Hébert commençait à voir le jour (vers les années 1940). la société québécoise ne s'était pas encore libérée des préjugés jansénistes s'attaquant à tout ce qui avait rapport avec la chair. Ce que Pierre 
Pagé écrit au sujet d'Agnès du Temps sauvage pourrait s'appliquer également à François Perreault, et a maints autres personnages de cette œuvre:

Agnès est marquée d'une culpabilité fondamentale: initiale, inséparablement liée a l'acte d'existence. Plus loin qu'elle-même, c'est en ses parents qu'elle était marquée (...) Du fond d'eux-mêmes, c'est la condition humaine qu'ils n'avaient pas acceptée, faite d'esprit, mais aussi de chair. Quelques générations leur avaient légué une vision janséniste de l'homme en lequel ils voyaient un ange déchu condamné à vivre dans un corps ${ }^{69}$.

Dans un tel milieu, comment l'homme ne se serait-il pas senti diminué par la présence du désir que le regard d'autrui découvre au fond de son être? Chez Anne Hébert, la clarté du jour illumine une passion qui, réfléchie par le miroir que constitue la société, est finalement percue par le sujet comme un mal. une profonde défaillance du corps et de l'âme. Et l'individu ploie sous le poids de cette condamnation. Sous l'emprise du regard de Claudine, elle-même influencée par l'opinion du village ${ }^{70}$. François demeure incapable de jouir de son corps, mème durant sa nuit de noces. L'état de sécheresse qui l'opprime semble naître surtout du refoulement d'un désir instinctif, jugé foncièrement mauvais :

J'abserve, dit-il, le couple étranger en sa nuit de noces. Je suis l'invité des noces (...)

J'éprouve une telle sécheresse. Ni désir, ni volupté. Sécheresse. Sécheresse de tout. Ainsi depuis toujours une volonté arbitraire a saccagé tout principe d'émotion et de jouissance en moi. Ah! ma mère, je ne pouvais deviner toute l'ampleur de votre destruction en moi! ${ }^{71}$

Si le désir est.le centre de la vie d'Élisabeth Rolland, c'est aussi ce dont, autant qu'Aurélie, elle se voit a jamais coupable: "Je n'ai jamais été innocente $\approx$, dit celle-ci. "Et Madame non plus»72. Dans la clarté ardente de la chambre où sa réalité intime lui revient déformée par le regard des témoins. la passion d'Élisabeth, juxtaposée au souvenir des malades du docteur Nelson, prend l'aspect d'une infirmité du corps et de l'âme. Ainsi la lumière hébertienne, en soumettant le désir de l'individu au regard et au jugement d'autrui, lui impose un verdict qui ne semble pouvoir être que négatif:

Ce désir absolu, dit Élisabeth (...)

Les malades et les infirmes vont faire irruption, nous saisir tous les deux. Nous dénoncer. Nous trainer sur la place publique. Nous livrer enchaînés à la justice. Un juge en perruque nous séparera l'un de l'autre. D'un seul coup d'épée. ${ }^{73}$

\section{De la fragmentation au désert du monde}

C'est ainsi que la lumière, les mains et le regard chez Anne Hébert conduisent graduellement au «désert du mondes: “Le jour se lève. Le désert 
du monde», dit Élisabeth ${ }^{74}$. Sans intuition ni tendresse, aiguë et tranchante, la clarté brutale qui domine au début l'univers du poète dessèche et fragmente la vie, le dissipant peu à peu. Aussi ne tardera-t-elle pas à anéantir complètement la réalité qu'elle éclaire.

C'est d'abord dans Le Torrent que nous pouvons mesurer tout l'effet destructeur d'une telle lumière. L'intensité du désir qui, dans la lumière du jour, amène François à fixer violemment la réalité, ne sait pourtant en dévoiler que des fragments; comme d'ailleurs le regard de Claudine perçoit plus clairement chacune des fautes de son fils ' a enregistrée minutieusement», que l'enfant lui-même ${ }^{75}$. Trop dure et perçante; cette vision, loin de découvrir la vie dans son intégrité et sa profondeur, ne fait que la dessécher et l'effriter:

Je touchais au monde par fragments, dit François ${ }^{76}$.

Le nom de Dieu est sec et s'effrite. Aucun Dieu n'habita jamais ce nom pour moi. Je n'ai connu que des signes vides. ${ }^{77}$

Analysant la structure romanesque du Torrent. Michèle Doray ecrit: "ll est intéressant de noter que Claudine se présente à François d'une manière disloquée: le héros ne perçoit d'elle que ses pieds, sa stature. ses mains ou bien le bas de son visage, mais il ne les perçoit pas simultanément" 78 . En effet, soumis à l'action d'une lumière aussi desséchante. François demeure incapable d'appréhender non seulement la personnalité intégrale de sa mère, mais aussi la totalité de son corps.

Dans le recueil Le Tombeau des rois, le poète, soumise à un éclairage semblable, se livre à la dislocation de son être le plus intime. Ici la fragmentation de la vie effectuée par la lumière et le regard prend tout son sens. Dans le poème "Inventairen, nous avons vu un soleil intense et une vision aiguë, associés à des mains tranchantes, percer et détruire le "mystère de la vie "elle-même, en morcelant le corps et l'âme. La vie du sujet y est "crevée " par le "fin couteau" du regard qui sépare impitoyablement le cour, le sang, les mains et la chevelure ${ }^{79}$. De même, la lumière opprimante du poème *Rouler dans des ravins de fatiguen, en perçant la poitrine du sujet, coupe tragiquement le coeur du corps, "l'agrafant périlleusement au cou, comme un "oiseau" susceptible de s'envoler d'un instant à l'autre ${ }^{80}$. Dans son article sur "La dislocation dans la poésie d'Anne Hébert», Gérard Bessette qualifie de masochiste une désagrégation aussi organique de la vie la plus intime:

Il faut entendre ici le mot a dislocation dans son acception la plus stricte, la plus anatomique: séparation des parties du corps. démembrement et, par voie de conséquence, tendance à considérer le corps comme un mécanisme mal monté, mal joint, enclin à la désagrégation : dont les différents organes n'obéissent pas à une volonté, à une impulsion centrale, mais agissent, bougent, se déplacent chacun pour soi, autonomes et chosifiés ${ }^{81}$.

Si c'est surtout dans les premières cuvres d'Anne Hébert que l'aspect destructeur et desséchant de la lumière et du regard a une valeur prédominante. 
la brutalité perçante de ceux-ci se retrouve ici et là dans ses ceuvres postérieures, et surtout dans Kamouraska, cuvre-synthèse de tout un cheminement poétique. Quand Elisabeth Rolland fixe intensément la maison de la rue Augusta, la lumière violente et “précise" qui éclaire cette demeure la défait petit à petit, en détaillant "chaque nervure 82 . Et plus le regard “jaune" de scour Julie viole les secrets de l'abbé Migneault, plus la dureté de cee regard ne livre à celui-ci, pour hanter son sommeil, qu'une image fragmentée de sa réalité, un portrait "rongé " et "déchiqueté»:

Il en vint à craindre toute rencontre avec sœur Julie, ne parvenant jamais a baisser les yeux avant que ne l'atteigne le regard jaune et moqueur au détour d'un corridor. Le pouvoir destructeur de sceur Julie agissait sur l'aumônier, sans rencontrer de résistance (...)

Quant a l'abbé, il eut des insomnies, des sueurs nocturnes et des cauchemars. Rongé par des insectes, broyé par des machines de fer et d'acier, déchiqueté, émietté, traité de raté par son père et de minable par sa mère (tous deux avaient des mâchoires et des écailles de crocodile), il finit par se rendre à l'évidence: sa parfaite nullité en ce monde ${ }^{83}$.

Il ne fait aucun doute que le "pouvoir destructeur" de sœur Julie est issu directement de ce regard dont la couleur nous rappelle trop vivement l'intensité de la lumière hébertienne. Alors que l'emploi de termes tels que "rongé". “broyé,, «machines de fer et d’acier», «mâchoires" et "écailles" sert ici à perpétuer le sème de dureté inhumaine, l'image des "insectes", rapprochée des participes "déchiqueté » et "émietté", traduit très bien la notion d'une vie pulvérisée, en passe de devenir cette "parfaite nulleté» qui clôture le texte.

Et c'est ainsi que, dans cette œuvre, le regard aigu lié à la violence du soleil nous mène graduellement à un désert, à un univers de sable, qui s'effrite, superficiel et nul: "Le pire qui pourrait encore marriver», affirme Élisabeth au lever du jour, “c'est d'être condamnée au désert du monde ${ }^{84}$. Percée jusque dans ses profondeurs et morcelée à l'infini, la réalité dévoilée par une telle lumière perd complètement son principe interne, se limitant à une surface. Dans un monde aussi brutalement éclairé, la vie est progressivement desséchée et amincie, voire anéantie.

L'image du sable prend ici toute sa signification. Si le sable définit si bien ce monde fragmenté et desséché par le soleil et l'acuité du regard, s'il se trouve autant à l'intérieur qu'à l'extérieur de l'être ${ }^{85}$, c'est qu'il est infiniment sec et morcelé, dur et irritant. C'est du sable que le cri d'une lumière opprimante, dans « Rouler dans des ravins de fatigue», semble verser dans l'oreille du sujet en la pénétrant ${ }^{85}$, et ce sont également des "doigts de sable» qui se tendent vers l'amante d' "Un bruit de soie», lorsque l'intensité de la lumière a dissipé la vie:

L'éclat de midi efface ta forme devant moi

(...)

Et découvre cette calme immobile distance 


\section{Entre tes doigts de sable et mes paumes} toutes fleuries ${ }^{87}$.

Et plus, dans Le Torrent. François fixe clairement le monde, plus il se soumet a l'emprise de la lumière, plus aussi sa voix sèche et grinçante l'associe a la dureté du sable: «Ma voix brève, rauque, m'était toujours pénible;, irritante à entendre ${ }^{88}$.

Morcelé et sec, le désert hébertien est réduit à unè surface plane, à une simple étendue. L'espace ensoleillé que l'auteur du poème "Inventaire" qualifie de "spacieux désert $\$ 89$ est fait de "scurires à fleur de peau " 90 , de jours aussi minces que des $\alpha$ rideaux ${ }^{99}$, de routes "sans âme» étendues au soleil ${ }^{92}$, enfin de tables "sans pieds», où le sujet dépose un visage «rongé " qui, dissocié de l'énigme du cceur, prend un aspect superficiel. Plus tard, "La ville tuée" de Mystère de la parole nous dépeint la sèche étendue d'un pays où on a banni toute émotion. Ici la lumière et la clairvoyance, la précision d'une vision aiguë, semblent "aplatir * la main, réduisant le corps a l'épaisseur de la peau ou de l'os. L'image externe du corps, sèchement percu, étouffe sa réalité intérieure. Les souvenirs, "passés au crible», perdent leur substance; amours et rèves disparaissent, tandis que l'espace horizontal de ce pays désertique efface toute profondeur:

La main droite de chacun fut posée à plat sur le ciel afin de vérifier chaque jointure et la précision des os dans la lumière

Les souvenirs furent passés au crible, tout amour impitoyablement saisi avec toute mémoire rêveuse ou insolente

Longtemps la douleur et la mort semblèrent subjuguées. Cela fit un beau pays sec pour s'étendre et faire le guet ${ }^{93}$.

Dans l'œuvre d'Anne Hébert, c'est finalement au vide absolu qu'aboutit l'éclat brutal du regard et de la lumière. Sous cette clarté intense et desséchante. la fragmentation progressive de la réalité est un anéantissement. Aussi le sujet se voit-il peu à peu dépossédé de son univers. La lumière intẹnse qui brille sur le domaine de Claudine aveugle de sa propre clarté: "J'ouvre les yeux sur un matin lumineux, dit François. Je suis face à face avec le matin. Je ne vois que le ciel qui $m$ 'aveugle ${ }^{94}$. Alors que le héros du Torrent regrette amèrement. l'inaccessibilité d'un monde que la violence du soleil, conjuguée au dur regard de sa mère, lui a dérobé ${ }^{95}$, l'amante du poème "Un bruit de soie " cherche en vain son amant dans la lumière de midi :

Tu trembles et luis comme un miroir

Tu m'offres le soleil à boire

A même ton visage absent ${ }^{96}$.

Le "visage absent» est métonymique ici de la perte totale de l'être. L'amant disparait intégralement sous l'éclat du jour, car le “miroir» qui le définit aux yeux du sujet n'existe qu'en fonction de ce qu'il reflète: la lumière elle-mème. Ainsi l'amante est-elle condamnée à un univers où seuls 
subsistent son propre regard et la puissance cosmique du soleil.

II n'est pas indifférent de noter une modification que subit maintenant la couleur dans l'univers poétique d'Anne Hébert. A mesure que s'intensifie la lumière et que celle-ci, conjointement avec le regard, morcelle. dessèche et anéantit le monde, le bleu et le jaune, teintes dominantes du jour hébertien, se transforment en blanc, leur clarté en dur éclat de miroir, et la vie pâlit sensiblement. Tel ce jardin de la rue Augusta, victime, lui aussi, de la violence destructrice de la lumière et du regard:

Depuis un instant, dit Ellizabeth, il y a quelque chose qui se passe du côté de la lumière. Une sorte d'éclat qui monte peu à peu et s'intensifie. Cela devient trop fort, presque brutal.

Soudain, cela s'arrête et se fixe sur une seule maison de briques rouges, angle des rues Philippe et Augusta. Isolée de ses voisines, par cette clarté qui lui tombe dessus, la maison se met à briller. Précise comme si on la regardait à la loupe. Lustrée. Emaillée de lumière. Le petit jardin pâlit sous un si grand soleil. Les hortensias bleus paraissent poudrées de blanc.

La couleur blanche, chez Anne Hébert, devient ainsi l'indice d'un monde en voie de disparition, progressivement rongé, desséché et vidé par la violence de la lumière et du regard. Les «filles bleues de l'été"98 et le ableu du ciel»

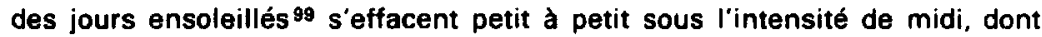
l'éclat blanc, vif et dur évoque le néant:

Tu trembles et luis comme un miroir

$(. .$.

Trop de lumière empêche de voir:

l'un et l'autre torche blanche

grand vide de midi ${ }^{100}$.

C'est ainsi directement au néant que mène la lumière intense qui prédomine dans les premières cuvres d'Anne Hébert. Captivante, oppressive et perçante, la clarté extraordinaire commune au regard du sujet, à ses mains qui la reflètent et à l'univers perçu, détruit progressivement ce qu'elle éclaire. par sa violence mème. Le paradoxe réside dans le fait que cette lumière insolite dont l'ardeur oriente l'individu vers autrui, rend en mème temps le monde inaccessible, en le desséchant et le morcellant à l'infini. It n'est pourtant guère possible à l'homme d'exister, dans l'cuure d'Anne Hébert, sans l'emprise d'une telle lumière. Si douloureuse que soit la violence de ce soleil associé au regard et aux mains, seule sa clarté paraît assurer la présence de la vie et le contact avec le réel, et seule elle maintient l'homme en communication avec la vie.

1. Anne Hébert, Mystère de la parole, Paris, Editions du Seuil, 1960, p. 69.

2. Anne Hébert, Les Songes en équilibre, Montréal, H.M.H., coll. L'Arbre, 1942, p. 63. 
3. Anne Hébert, Kamouraska, Paris, Editions du Seuil, 1970, pp. 146-147.

4. Ibid., p. 107.

5. Anne Hébert, Poèmes, Paris, Éditions du Seuil, 1960, p. 59: «J'ai mon cœur au poing/ Comme un faucon aveugle."

6. aLa ville tuée», Mystère de la parole. Paris, Editions du Seuil, 1960. pp. 94-95.

7. «Rouler dans des ravins de fatigue»: Le Tombeau des rois, Québec, Institut littéraire du Québec, 1953, p. 55.

8. P.-H. Lemieux, Entre songe et parole, Ottawa, Editions de l'Université d'Ottawa, 1978, pp. 178-180.

9. «Un simple éloge de l'innocencen, interview par Réginald Martel, La Presse, ler décembre 1979: «On dit souvent «je w ou a nous dans les poèmes. Je voulais dire aussi $\propto$ il $»$, «elle» et a eux». Je voulais peut-être prendre une distance par rapport au moi.*

10. Voir Les Songes en équilibre, Montréal, H.M.H., coll. L'Arbre, 1942, p. 69 a J'étouffe dans un jardin/ Des bouquets/.../M'écoeurent et m'enfièvrent/ Jardin clos», cf. Le Torrent, Montréal, Editions Beauchemin, 1950. p. 64 : « Je ne puis imaginer ma fin en dehors de moi (...) Je joue, éveillé, avec les éléments d'une fiève qui m'apaisen.

11. Anne Hébert, Le Torrent, Montréal, Éditions Beauchemin, 1950, p. 38.

12. A. Legrand, Anne Hébert: Français 341. Montréal, Librairie de I'Université de Montréal, 1973, p. 124.

13. Anne Hébert, Le Torrent, Montréal, Editions Beauchemin, 1950, pp. 51-52 Souligné dans le texte.

14. Anne Hébert, Les Enfants du sabbat, Paris, Editıons du Seuil, 1975, p. 9.1.

15. Anne Hébert, Le Torrent, Montréal, Editions Beauchemin, 1950, pp. 18-19.

16. Ibid., p. 50.

17. Voir: Kamouraska, Paris, Editions du Seuil, 1970, pp. 146-147. Texte cité plus haut, note 3.

18. Ibid., p. 147.

19. Ibid. pp. 103-104.

20. Anne Hébert, Le Torrent, Montréal, Éditions Beauchemin, 1950, p. 19.

21. Anne Hébert, Les Songes en équilibre, Montréal, H.M.H., coll. L'Arbre. 1942. p. 25.

22. "La robe coraii", in Le Torrent, p. 110.

23. A. Juéry, CEuvres en prose d'Anne Hebert, Ottawa. Ph. D. Université d'Ottawa. 1977, p. 19.

24. Le Torrent, p. 14.

25. Ibid., p. 39.

26. Ibid. p. 15.

27. Albert Legrand, op. cit., p. 117.

28. "Jour de juin", in Les Songes en équilibre, Montréal, H.M.H., coll. L'Arbre, 1942, p. 13.

29. Le Torrent. p. 39.

30. Pierre Pagé, Anne Hébert, Montréal, Éditions Fidès, 1965, p. 92. Cf. J.-C. Godin, "Rebirth in the world", Yale french Studies, $n^{\circ} 45,1979$, p. 144.

31. L'ange de Dominique $*$, in Le Torrent, 2ème édition, p. 79.

32. Ibid., p. 68.

33. Ibid., pp. 104-105.

34. "L'Infante ne danse plus", Gants du ciel, $n^{\circ} 4$, juin 1944. p. 5.

35. A. Élizabeth. L'Arche de midi, p. 31.

36. F. Macri, L'Aliénation dans l'ceuvre d'Anne Hébert, Sherbrooke, M.A. Université de Sherbrooke, 1977, p. 108. 
37. "Les mains $*$, Le Tombeau des rois, pp. 21-22.

38. Pierre-Hervé Lemieux, op. cit., pp. 42-43.

39. «Eternité», Les Songes en équilibre, Montréal, H.M.H., coll. L'Arbre, 1942, p. 42.

40. Anne Hébert. Les Chambres de bois. Paris, Editions du Seuil, 1958, p. 129.

41. Un bruit de soie», Le Tombeau des rois, pp. 57-58.

42. Pierre-Hervé Lemieux, op. cit., p. 192.

43. Anne Hébert, Les Chambres de bois, p. 69.

44. "Le silence", Le Figaro littéraire, 10 janvier 1971, p. 25.

45. Jean-Louis Major, Anne Hébert et le miracle de la parole, Montréal, Presses de I'Université de Montréal, 1976, p. 14. Cf. N. Bishop, op. cit., pp. 196-201.

46. «Rouler dans des ravins de fatiguex, in Le Tombeau des rois, p. 55.

47. a La robe corails, in Le Torrent, 2 ème édition, p. 118.

48. Kamouraska, p. 100.

49. Ibid., p. 49.

50. Ibid., p. 100.

51. L. Lefebvre, Le Theme de la femme dans l"ceuvre romanesque d'Anne Hébert. Trois-Rivieres, M.A., UQTR, 1976, p. 136

52. \&Inventare», Le Tombeau des rois. p. 29.

53. Ibid., pp. 29-30.

54. Ici, nous nous écartons du jugement de Pierre-Hervé Lemieux qui écrit: “Rien n'est encore déterré de l'enfance et des profondeurs du ccourn, op. cit., p. 78.

55. Voir « inventaire $*$, Le Tombeau des rois, p. 30.

56. Anne Hébert; a La mercière assassinée », Le Temps sauvage suivi de La Mercière assassinée et Les Invités au procès. Montréal, H.M.H., coll. L'Arbre, 1967, pp. 146 et 149.

57. Les Chambres de bois, p. 95.

58. Le Torrent, pp. 50-52.

59. Ibid., p. 59.

60. Ibid., p. 59.

61. Kamouraska, pp. 41.42 .

62. Ibid., p. 71.

63. Le Torrent, pp. 62-63.

64. Kamouraska, p. 142.

65. Ibid., p. 103.

66. M. Telles, Lecture d'Anne Hébert: Kamouraska. Montréal. M.A., McGill University. 1970, p. 57.

67. Voir Le Torrent, p. 47.

68. Les Enfants du sabbat, pp. 72-73.

69. Pierre Pagé, op. cit., p. 83.

70. Le Torrent, p. 19.

71. Ibid. p. 49.

72. Kamouraska, p. 61.

73. Ibid., pp. 142-143.

74. Ibid., p. 160.

75. Le Torrent, p. 11.

76. lbid., p. 9.

77. Ibid., p. 36.

78. M. Doray, Le Torrent ou le mythe devenu roman, Montréal, M.A., McGill University, 1973, p. 58. 
79. anventaire ., Le Tombeau des rois, pp. 29-30.

80. «Rouler dans les ravins de la fatigue», Le Tombeau des rois, p. 55.

81. Gérard Bessette, a La dislocation daris la poésie d'Anne Hébert», Une littérature en ébullition. Montréal, Editions du Jour, p. 13.

82. Kamouraska, p. 50.

83. Les Enfants du sabbat, p. 53.

84. Kamouraska, p. 160.

85. Voir supra.

86. "Rouler dans des ravins de fatigue», Le Tombeau des rois, p. 55.

87. «Un bruit de soie», Le Tombeau des rois, p. 57.

88. Le Torrent, p. 26.

89. Le Tombeau des rois, p. 30.

90. "L'envers du monde", Le Tombeau des rois, p. 52.

91. Un bruit de soiè, Le Tombeau des rois. p. 58: a Mes mains écartent le jour comme un rideau."

92. Le Torrent, p. 15.

93. "La-ville tuéen. Mystère de la parole, pp. 94-95.

94. Le Torrent, p. 36.

95. Ibid., p. 9.

96. «Un bruit de soie», Le Tombeau des rois, p. 57.

97. Kamouraska, p. 50.

98. L'envers du monde., Le Tombeau des rois, p. 52.

99. "Rouler dans des ravins de fatigue», ibid., p. 55: «Bleu du ciel/ Grand cri de la lumière au-dessus de nousx.

100. "Un bruit de soie», ibid., p. 57. 